\title{
Manipulation of a7 nicotinic acetylcholine receptor signaling in primary ovine fetal microglia cultures
}

\section{Martin Frasch ( $\square$ mfrasch@uw.edu )}

University of Washington

\section{Mingju Cao}

McGill University

Marina Cortes

Hai Lun Liu

McGill University

\section{Method Article}

Keywords: CHRNA7, microglia, fetus, sheep, RNAseq, inflammation

Posted Date: February 1st, 2018

DOl: https://doi.org/10.1038/protex.2018.013

License: (c) (1) This work is licensed under a Creative Commons Attribution 4.0 International License.

Read Full License 


\section{Abstract}

Neuroinflammation in utero may result in life-long neurological disabilities. Microglia play a pivotal role, but the mechanisms are poorly understood. No early postnatal treatment strategies exist to enhance neuroprotective potential of microglia. An important step toward that goal is establishing adequate pathophysiological models where microglia function in a large, well gyrated mammalian brain can be studied. Here we present the protocol to manipulate ovine fetal microglia in primary cultures in vitro without or with previous exposure to endotoxin \(lipopolyssacharide, LPS) in vivo. We present the approach of blocking or stimulating the $a 7$ nicotinic acetylcholine receptor $\backslash(a 7 n A C h R)$ in fetal microglia which allows to enhance or reduce their pro-inflammatory phenotype when incubated with LPS. The measurement of cytokine IL-1beta in supernatant and the RNAseq pipeline are presented.

\section{Introduction}

Fetal inflammation contributes significantly to the aetiology of the perinatal brain injury with potentially life-lasting neurological. especially cognitive deficits. Second-hit hypothesis states that postnatal inflammatory insults may enhance antenatally occurring subclinical infections, thus further exacerbating the brain injury. No treatment exists. a7 nicotinic acetylcholine receptor $\backslash(a 7 n A C h R)$ signaling in microglia modulates their inflammatory phenotype. Here we present the experimental protocol to create a robust animal model in pregnant sheep that addresses the above-stated need.

\section{Reagents}

Animals: Pregnant ewes Cell culture - LPS \(100ng/ml, Sigma L5024, from E coli 0127, B8) - aBungarotoxin \(Tocris Bioscience Cat\# 2133), a selective a7nAChR antagonist - AR-R17779 hydrochloride I(Tocris Bioscience Cat\# 3964), a selective a7nAChR agonist IL-1 beta cytokine measurements in vitro RNAseq protocol - TRIzol Reagent $\backslash$ (Life Technologies) - Illumina TruSeq RNA Sample Preparation v2 kit $\backslash($ Illumina)

\section{Equipment}

RNAseq - RNA Nano Chips \(Agilent RNA 6000 Nano Chips) with Agilent 2100 BioAnalyzer - Illumina HiSeq2500

\section{Procedure}

**In vitro microglia culture protocol** Obtain the fetal sheep brain tissues during sheep autopsy after completion of the in vivo experiment to conduct the in vitro study. Fetal sheep microglia culture protocol was adapted from an established human adult and fetal microglia culture protocol that was modified to include a myelin removal step following the high-speed centrifugation. Fetal sheep cells were plated on poly-L-lysine $\backslash(P L L)$-coated tissue culture flasks at a concentration of $2 \times 10^{6}$ cells $/ \mathrm{ml}$ in DMEM with $5 \%$ 
heat-inactivated fetal bovine serum \(Gibco, Canada Origin), 1\% penicillin/ streptomycin, and 1\% glutamine $\backslash(5 \%$ DMEM), in which microglia grow best. 56 Cells were allowed to incubate for seven days at $37^{\circ} \mathrm{C}, 5 \% \mathrm{CO}_{2}$, followed by a media change by centrifugation and the addition of re-suspended cells back to the culture flask. Cells were continued to incubate for seven more days with $5 \%$ DMEM at $37^{\circ} \mathrm{C}, 5 \%$ $\mathrm{CO}_{2}$, before the floating cells were collected. After carefully collecting the floating microglia to avoid contamination with astrocytes and oligodendrocytes, the cells were incubated in 24-well plate at $1 \times 10^{5}$ cells $/ \mathrm{mL}$ with $5 \%$ DMEM for another 4-5 days, and then treated with or without LPS $\backslash(100 \mathrm{ng} / \mathrm{ml}$, Sigma L5024, from E coli 0127, B8) for $6 \mathrm{~h}$. Cell conditioned media were collected for cytokine analysis, $0.5 \mathrm{ml}$ TriZol per well added for RNA extraction. To verify microglia purity, a portion of floating cells was cultured in 24-well plate under the above conditions for flow cytometry analysis $\backslash$ (see below). The cell morphology was documented with light microscopy. Another portion of floating cells was plated onto Lab-Tek 8 well chamber glass slide $\backslash$ (Thermo Scientific) and treated with or without LPS for immunocytochemistry analysis. **RNAseq approach ${ }^{\star \star}$ The overall experimental design was divided into three phases: sequencing, quantification and discovery $\backslash($ Figure $1 \mathrm{~A})$. RNA extraction and RNA quantification: Total RNA was extracted from cultured microglia using TRIzol Reagent $\backslash$ (Life Technologies). RNA quantity and quality $\backslash$ (RNA integrity number, RIN) was established by using a RNA Nano Chip \(Agilent RNA 6000 Nano Chips) with Agilent 2100 BioAnalyzer. RNAseq libraries were prepared using Illumina TruSeq RNA Sample Preparation v2 kit \(Illumina) and quality control was performed on the BioAnalyzer. Single-end 50-bp sequencing was performed at high throughput on an Illumina HiSeq2500 at the CHU Ste-Justine Core Facility Sequencing Platform.

\section{Timing}

Tissue processing and cell plating: $\sim 6 \mathrm{~h}$ Cell culture: microglia take 2 weeks from day 0; astrocytes take 4 weeks $\backslash$ (or additional 2 weeks after microglia). In vitro treatment experiment: depends on your objectives; LPS model we described takes $\sim 7 \mathrm{~h}$.

\section{Troubleshooting}

TBD

\section{Anticipated Results}

This depends on the treatment you choose to perform in vitro. Expect 95\% purified microglia and astrocytes that respond well to LPS and are manipulated further by alpha7 nAChR drugs.

\section{References}

We associated three publications that describe all experiments in detail.

\section{Acknowledgements}


Department of Clinical Sciences at Universite de Montreal and CRRA \(Centre de Recherche en Reproduction Animale), both in St-Hyacinthe, QC. In particular, huge thanks to Profs. Andre Desrochers, Gilles Fecteau and Patrick Burns. Also big thanks to the tireless graduate students Dr. Daniel Durosier and Ms. Hai Lun Liu. Special thanks to Dr. Jack Antel's lab at Montreal Neurological Institute \(MNI) at McGill University who encouraged, trained and supported us every step of the way to bring this project and approach to fruition.

\section{Figures}

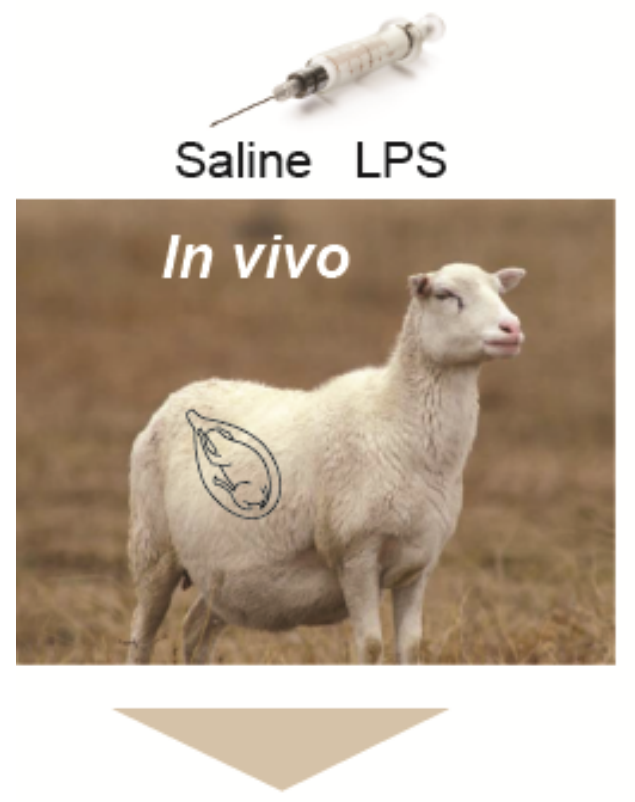

In vitro

Experi-

Fetal Brain Control mental Microglia:

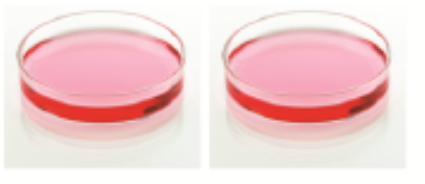

\section{SEQUENCE}

Sequence at high throughput (Illumina HiSeq2500)

Read prep, mapping to Ovis aris v3.1 (Tophat2)

Read count (HTseq)

\section{QUANTIFY}

Differential analysis (DESeq2)

Differentially expressed $\wedge$ and $\boldsymbol{\Downarrow}$ regulated genes
Genes clustering (ToppCluster) into heatmaps

Conversion to Human Orthologs,

Gene Ontology (Gorilla), Protein-protein network analysis (String-db), Reactome analysis

\section{Figure 1}

Protocol overview In vivo - in vitro model of LPS double-hit. The effects of the two-hit endotoxin exposure on neuroinflammation in vivo and in vitro are measured in this fetal sheep model of an asymptomatic fetal infection. The primary microglia cultures are derived over a period of two weeks and incubated with 
LPS for 6 hours preceded by a pre-treatment with the cholinergic agonist or antagonist. IL-1beta levels in the supernatant are determined by a commercial ELISA and RNAseq is conducted to quantify the microglial transcriptome depending on the treatment. 